\title{
DISTAL ARTHROGRYPOSIS TYPE IIB IN A GIRL: AUTOSOMAL RECESSIVE INHERITANCE?
}

\author{
Masato TsuKaHARA and Tadashi KAJI \\ Department of Pediatrics, Yamaguchi University School of Medicine, \\ Ube, Yamaguchi 755, Japan
}

\begin{abstract}
Summary A 2-year-old girl with distal arthrogryposis type IIB is dèscribed. She is mental retarded, has congenital contractures of the hands and feet, ulnar deviation of the fingers, short stature, ptosis, blephalophimosis, epicanthal folds, large prominent ears, a short neck and abnormal dermatoglyphic patterns. In addition, she shows several clinical features not characteristic of the syndrome. These features include delayed osseous maturation, a preauricular pit, a narrow and high-arched palate, pectus carinatum, a stiff linea alba, and a sacral dimple. Her parents, both healthy, were first cousins, of each other. Thus the disease in the patient either may represent a fresh mutation of an autosomal dominant disease, or may be inherited as an autosomal recessive trait.
\end{abstract}

\section{INTRODUCTION}

Distal arthrogryposes, congenital contractures of the hands and feet, are classified into two groups: type I, with distal limb contractures, and type II, with distal limb contractures and additional manifestations (Hall et al., 1982). Distal arthrogryposis type II is further divided into five subgroups, IIA through E, according to the respective additional cardinal malformations, such as cleft palate and short stature (IIA), ptosis (IIB), cleft lip and palate (IIC), scoliosis (IID) and trismus (IIE). Type IIB is characterized by short stature, an unusual facial appearance with ptosis, epicanthal folds, poorly formed, large anteverted ears, a short neck, and very smooth, shiny, tapering fingers with mild camptodactyly with ulnar deviation. Apparent autosomal dominant inheritance, with or without keratoconus, decreased facial expression, and decreased ocular range of motion also mark the type IIB patient.

Received September 22, 1984 


\section{CASE REPORT}

A $21 / 6$ year-old girl was born in a breech position after a 40 week pregnancy to a 22-year-old, primigravida mother and a 27-year-old father, both healthy. The parents are first cousins (Fig. 1). The parents live on Mishima, an island with a population of 1,500 in the Sea of Japan, $44 \mathrm{~km}$ north of the northern coast of Yama-

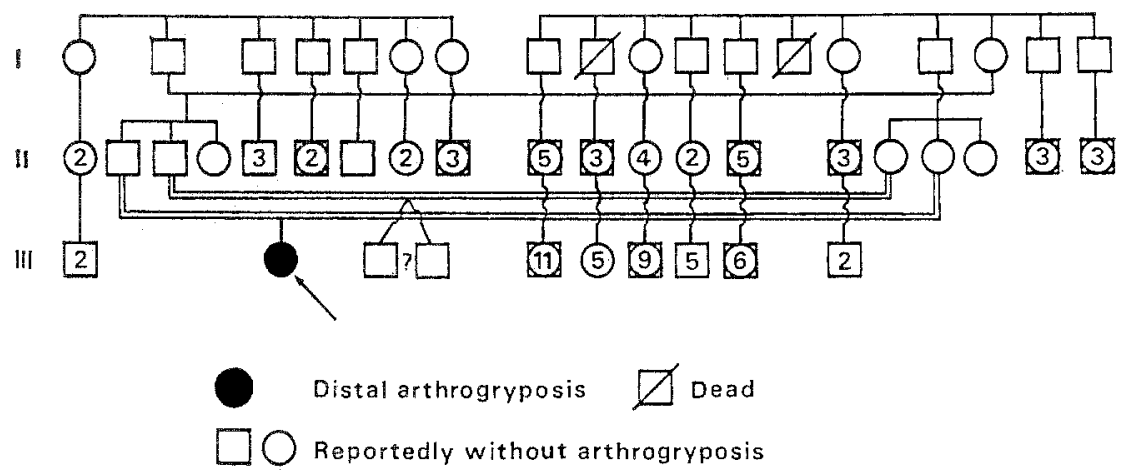

Fig. 1. Family tree. The parents of the patient are first cousins.

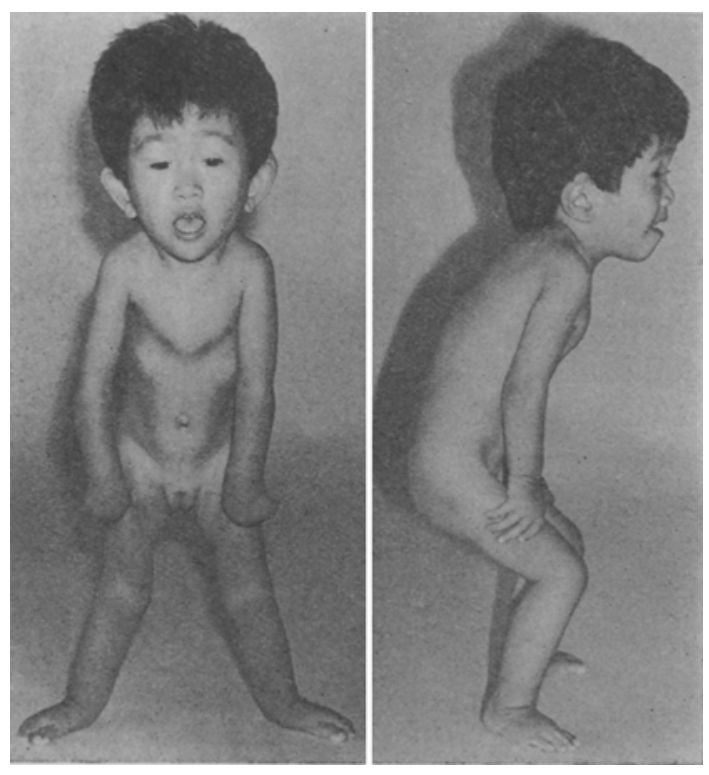

Fig. 2. The patient at age $21 / 6$ years. Note the large, prominent ears, ptosis, open mouth, limited facial expression and short neck. Contractures at elbows, wrists, hips, knees, ankles, and hands. 
guchi Prefecture. The residents of the island are highly inbred. There is no family history of malformations. Fetal movement during pregnancy was feeble. At birth, the patient weighed $2,400 \mathrm{~g}(-1.7 \mathrm{SD})$, measured $45 \mathrm{~cm}(-2.2 \mathrm{SD})$ and her head circumferance was $34 \mathrm{~cm}(+0.6 \mathrm{SD})$. Contractures of distal joints, including the hands and feet, were noted. When seen by us at 26 months of age, she weighed $6,650 \mathrm{~g}(-3.9 \mathrm{SD})$, her height was $72.3 \mathrm{~cm}(-4.1 \mathrm{SD})$, and her head circumferance was $47.2 \mathrm{~cm}(-0.4 \mathrm{SD})$. Her general features were apelike, as she had slightly flexed elbows, hip- and knee-joints (Fig. 2). Her shoulders, elbows, wrists, hips, knees and ankles exhibited a decreased range of motion. Other features noted included camptodactyly and ulnar deviation of the fingers at the metacarpophalangeal joints, smooth tapering fingers with absent interphalangeal creases (Fig. 3a), pes equinovarus, contractures of the distal phalangeal joints of the feet, and medially deviated toes. Craniofacial abnormalities noted on admission included a masklike face, blephalophimosis (the palpebral fissure length; rt $2.0 \mathrm{~cm} ; 1 \mathrm{t} 2.1 \mathrm{~cm}$ ), bilateral ptosis, long eyelashes, epicanthal folds, large, simple, anteverted and low-set ears, a right preauricular pit, a narrow and high-arched palate, an open mouth and prognathism (Fig. 2). Also noted were a short neck, pectus carinatum, stiff linea alba, iliac and coccygeal dimples (Fig. 3c) and deep plantar furrow (Fig. 3d). Neurological examination revealed an absence of biceps, triceps, knee jerks and normal

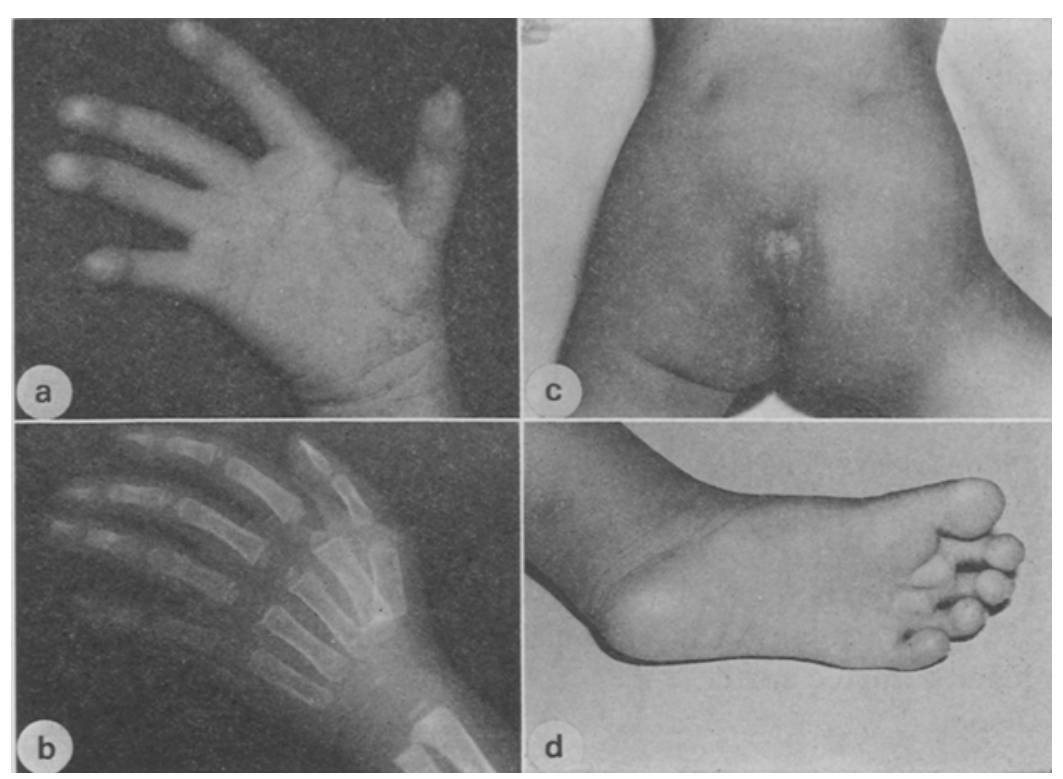

Fig. 3. a: The right hand with camptodactyly, ulnar deviation of the fingers, smooth fingertips, absent interphalangeal creases and hypoplastic dermal ridges. $\quad b$ : The right hand with a carpal bone at the age of 6 months. c: Dimpling at the hip and coccyx. d: The left foot with deep plantar furrows. 

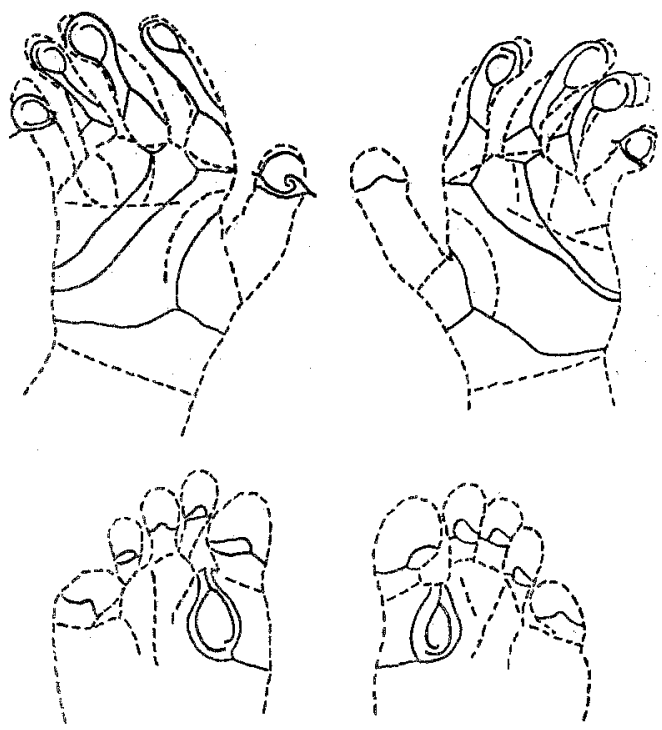

Fig, 4. Dermatoglyphic patterns of the palms and soles.

ankle jerks. She spoke several words, but no meaningful sentences. She has not been toilet trained. Her developmental quotient has been estimated to be at 69 using the Tsumori and Inage Scale of Developmental Maturity (1977). Fundoscopic examination revealed no abnormalities.

Laboratory data. The serum creatine phosphokinase, aldolase, and electrolyte levels were normal. G-banding chromosome analysis revealed a 46,XX karyotype.

$X$-ray examination. Complete skeletal survey at 26 months revealed slight digital markings of the skull and kyphosis of the thoracic vertebral column. Of the carpal bones only the capitate was ossified (Fig. 2b), with its degree of maturity corresponding to the 6-month-level (Sugiura and Nakazawa, 1979). The multiple center method of Elgenmark (1946) showed development comparable to that of a 15 month old infant. A CT scan revealed no abnormal findings.

Dermatoglyphics (Fig. 4). Dermatoglyphic studies revealed hypoplastic dermal ridges and simple ridge patterns on the palms and finger tips, and an absence of interphalangeal creases on both hands. Left fingers: double loop, whorl (w), w, w, w; right fingers: arch, w, w, w, w. The total finger ridge count was 167 . Her abnormal palmar patterns included radially deviated axial triradii, and an absence of interdigital triradii $\mathrm{c}$ and $\mathrm{d}$.

\section{DISCUSSION}

Our patient has clinical features characteristic of contracture of the hands and feet and ulnar deviation of the fingers, with lesser contractures or limitations of 
movement in the shoulders, elbows, wrists, hips, knees, and the ankles. She also shows mental retardation, short stature and craniofacial abnormalities including a mask-like face, ptosis, blephalophimosis, epicanthal folds, large prominent ears, a short neck and abnormal dermal patterns (Hall et al., 1982). In addition, she exhibits several clinical features not described previously in patients with the disease. These features include delayed osseous maturation, a preauricular pit, a narrow and high-arched palate, pectus carinatum, stiff linea alba, and a sacral dimple.

Hall et al. (1982) cited eight patients who fit the diagnosis of arthrogryposis type IIB, three of whom were of their own patients and five whose case were documented in the literature. Of these, five came from two families: a family with an affected father, daughter and son (Aase and Smith, 1968) and another family with an affected mother and daughter (Hall et al., 1982). The patient reported by Bijlsma (1980), an 8-year-old boy, had a mother with a cleft lip and a father with camptodactyly. The remaining two patients were sporadic cases of arthrogryposis type ШB (Krieger and Espiritu, 1972; Hall et al., 1982). There were no instances of consanguinity. Thus, it seems that the disease is inherited as an autosomal dominant trait, the sporadic cases representing a fresh mutation. Our patient may also represent a fresh mutation, although the father was relatively young, age 27, years when the patient was born. Alternatively, her disease may have been transmitted in an autosomal recessive fashion, in view of the fact that her parents were first cousins. Further reports of similar families may help to clarify the issue.

\section{REFERENCES}

Aase, J.M. and Smith, D.W. 1968. Dysmorphogenesis of joints, brain, and palate: A new dominantly inherited syndrome. J. Pediatr. 73: 606-609.

Bijlsma, J.B. 1980. Case report 60-cleft palate, flexion contracture, aqueductal stenosis, and mental retardation. Syndrome Identification IV (1): 4-5.

Hall, J.G., Reed, S.G., and Greene, G. 1982. The distal arthrogryposis: Delineation of new entities-review and nosologic discussion. Am. J. Med. Genet. 11: 185-239.

Krieger, I. and Espiritu, C.E. 1972. Arthrogryposis multiplex congenita and Turner phenotype. Am. J. Dis. Child. 123: 141-144.

Sugiura, Y, and Nakazawa, O. 1979. Bone Age. X-ray Diagnosis of Bone Maturation (in Japanese), Chugai Igaku Co., Tokyo

Tsumori, M. and Inage, N. 1977. Developmental Screening Test for Infants (in Japanese), Dainippon-Tosho Co., Tokyo 